\title{
Valorando los programas de apoyo al emprendedor en las incubadoras de empresas en Cataluña
}

\author{
Cerdán-Chiscano, Mónica* \\ Jiménez-Zarco, Ana Isabel** \\ Torrent-Sellens, Joan***
}

\section{Resumen}

A fin de apoyar de forma efectiva a los emprendedores, las incubadoras desarrollan una oferta de programas de soporte a la creación de empresas acorde a las necesidades de las que resultan acogidas. La supervivencia y el éxito de las incubadoras dependen de la valoración recibida por estos programas, de tal modo que identificar los factores influyentes en la valoración realizada por emprendedores será clave en su proceso de gestión estratégica. El análisis de una muestra de 29 microempresas instaladas en diferentes incubadoras de Cataluña (España), evidencia la existencia de ciertos factores que influyen notablemente en la valoración de los diferentes programas de apoyo ofrecidos. Su análisis también permite establecer la existencia de diferencias importantes en los niveles de significación de cada factor, en función del grado de especialización del programa evaluado. Un incremento en el grado de orientación hacia el mercado, una especialización su oferta de programas de apoyo atendiendo al tipo de empresa y al perfil del

\section{Recibido: 23-04-12. Aceptado: 02-07-13}

* Doctora en Organización de empresas, Universidad Politécnica de Cataluña (UPC). Licenciada en Investigación y Técnicas de Mercados, Universidad de Barcelona (UB). Innovation and Marketing Manager del Parc de Recerca UAB (http://www.uab.es) e investigadora asociada del grupo interdisciplinario de investigación sobre las TIC, i2TIC (http:// i2tic.net/ en/people/monica-cerdan-chiscano/).

** Doctora en Ciencias Económicas y Empresariales, Universidad de Castilla-La Mancha, (UCLM); Post-grado en la construcción de modelos en Ecología y Manejo de Recursos Naturales de la Universidad Politécnica de Cataluña (UPC). Profesora Agregada del Área de innovación y marketing en los Estudios de Economía y Empresa y en la UOC Business School, Universitat Oberta de Catalunya, e investigadora del grupo interdisciplinario de investigación sobre las TIC, (i2TIC) http://i2tic.net/es/equipo/ana-isabel-jimenez/).

*** Doctor en sociedad de la información y el conocimiento, Universidad Oberta de Cataluña (UOC). Master en Economía Aplicada y Licenciado en Ciencias Económicas y Empresariales por la Universidad Autónoma de Barcelona (UAB). Profesor de los Estudios de Economía y Empresa, Director del grupo interdisciplinario de investigación sobre las TIC, i2TIC (http://i2tic.net/en/people/joan-torrent-sellens/), y director de la UOC Business School. 
emprendedor, y el establecimiento de una estrecha relación entre la institución y las redes de emprendedores, son algunas de las formulas estratégicas recomendadas para garantizar la supervivencia y la sostenibilidad de la incubadora a través del tiempo.

Palabras clave: Creación de empresas, incubación de empresas, innovación, organización institucional, emprendedor.

\title{
Evaluating Entrepreneur Support Programs at Business Incubators in Cataluña
}

\begin{abstract}
In order to support entrepreneurs effectively, incubators develop support programs for creating enterprises according to the needs of those that recur to them. The survival and success of the incubators depends on the evaluation received regarding these programs. Therefore, identifying the factors that influence evaluations made by the entrepreneurs would be key in the incubators' strategic management process. Analysis of a sample of 29 micro-enterprises installed at different incubators in Cataluña (Spain) demonstrate the existence of certain factors that influence notably the evaluation of the different support programs offered. This analysis also permits establishing the existence of significant differences in the signification levels for each factor, related to the degree of specialization for the evaluated program. An increase in the degree of market orientation, a specialization in their offer of support programs according to the type of company and the profile of the entrepreneur and the establishment of a close relationship between the institution and networks of entrepreneurs are some of the strategic formulas recommended to guarantee the survival and sustainability of the incubator over time.
\end{abstract}

Keywords: Creation of enterprises, incubation of enterprises, innovation, institutional organization, entrepreneur.

\section{Introducción}

Hoy día no se cuestiona, que la creación de nuevas empresas constituye una fuente de creación de empleo y desarrollo local (Birch, 1979), así como tampoco se cuestiona que el empleo de mecanismo de apoyo al emprendimiento, ejerce un efecto positivo tanto en el número de empresas creadas como en las tasas de supervivencia (Allen, 1985; 1990; Alonso, 2007; Peterson, 1985; Smilor, 1986).

Diversos trabajos señalan como la creación de nuevas empresas no solo depende de factores tangibles (capital) o físicos, sino especialmente de factores intangibles (socioculturales, estructura ins- titucional). En este sentido, el papel de las incubadoras cobra especial importancia, afectando de forma directa a la generación de actividad en el territorio. Es por ello que desde los ámbitos políticos y administrativos se impulsa la creación de instrumentos de soporte a nuevas iniciativas de empresas en los territorios, proliferando de esta forma las incubadoras de empresas.

Las incubadoras, ejercen un papel fundamental en este proceso, por cuanto ponen al alcance de los emprendedores algunos de los instrumentos y servicios necesarios para acelerar el crecimiento y éxito de los proyectos empresariales. Sin embargo, algunas investigaciones sugie- 
Valorando los programas de apoyo al emprendedor en las incubadoras de empresas... Cerdán-Chiscano, Mónica; Jiménez-Zarco, Ana Isabel y Torrent-Sellens, Joan

ren que las incubadoras podrían mantener vivas durante largos períodos de tiempo a empresas enfermizas, que no podrían subsistir fuera de este entorno protegido (Nueno, 1996). Es en este contexto donde la literatura (Allen, 1985; 1990; Alonso, 2007; Peterson, 1985; Smilor, 1986) sugiere la necesidad de valorar el rol y los resultados de las incubadoras de empresas, para que estas sean capaces de diseñar programas de acompañamiento eficaces en el soporte de creación de empresas innovadoras y se conviertan en un eslabón más de la cadena de agentes institucionales y económicos del fomento al desarrollo económico local. No obstante, faltan estudios empíricos que expliquen los mecanismos de apoyo ofrecidos por las incubadoras de empresas desde el punto de vista de la demanda.

Considerando esta necesidad, el presente trabajo tiene como objetivo analizar cómo algunos factores relativos del emprendedor y la empresa influyen en la valoración realizada sobre los diferentes programas de apoyo ofrecidos por la incubadora de empresas. A partir de una muestra de 29 microempresas instaladas en diferentes incubadoras catalanas, el empleo de técnicas estadísticas no paramétricas señalan la significatividad de variables como el perfil del emprendedor, o características estructurales de la empresa -dimensión, facturación y carácter innovador - sobre la valoración recibida por los diferentes programas de apoyo ofrecidos por la incubadora. Una vez presentadas las conclusiones y limitaciones, este trabajo finaliza ofreciendo algunas recomendaciones de interés para la gestión estratégica de las incubadoras.

\section{El origen de las incubadoras de empresas}

Desde la década de 1980, diversas instituciones públicas o privadas vienen desarrollando programas, proyectos que impulsan la creación y el crecimiento de las empresas de su ámbito de influencia. Entre ellos cabe destacar el fenómeno de las incubadoras de empresas. El rápido desarrollo alcanzado por esta modalidad de apoyo empresarial ha sugerido una importante evolución de su definición. En sus inicios, las incubadoras de empresas eran promovidas por la administración local o autonómica, con el fin de fomentar la aparición de emprendedores y la creación de puestos de trabajo para el desarrollo económico local. Actualmente, las incubadoras pueden ser promovidas por instituciones de diversa naturaleza (públicas-privadas) así como estar especializadas en empresas de determinados sectores, pero en general su misión fundacional se mantiene. Constituyen un servicio local que proporciona la infraestructura y los servicios de apoyo a la creación y consolidación de nuevas empresas; aunque las condiciones de acceso son restrictivas, una vez aceptados los proyectos, las empresas de nueva creación tienen acceso rápido a determinados recursos costosos y difíciles de acceder en circunstancias normales.

Pese al papel jugado por las incubadoras como instrumentos que favorecen la iniciativa y el crecimiento empresarial (Allen y McCluskey, 1990), trabajos como los de Nueno (1996) y Alonso y Fageda (2007) sugieren que la labor de las incubadoras podría tener un efecto pernicioso sobre algunas empresas. En su opi- 
nión, en un entorno protegido donde se ofrecen diferentes programas y servicios de apoyo, resulta fácil mantener con vida durante largos períodos de tiempo a empresas enfermizas que en otras circunstancias no podrían sobrevivir.

\section{Programas de apoyo al emprendedor}

Catalunya constituye una comunidad autónoma española pionera en la creación y establecimiento de programas de apoyo al emprendedor. De ahí el interés de su análisis.

En el caso concreto de Cataluña, la oferta de de programas de soporte al emprendedor se caracteriza por una amplia oferta tanto en lo referente al número de instituciones públicas que ofrecen los servicios, como por la variedad de programas existentes (Urbano y Veciana, 2001).

Asimismo, de acuerdo a Urbano y Veciana (2001), el emprendedor suele recibir los servicios de soporte a la creación de empresas en la Administración Autonómica y local de Cataluña. Es en este contexto, de incubadora dependiente de la Administración Local, es donde se centra esta investigación.

Los programas de soporte a la creación de empresas en las incubadoras de Cataluña, se basan en medidas de soporte no económicas: información y orientación, asesoramiento, formación, seguimiento e incubación, y en medidas de soporte económicas: acceso a ayudas financieras y capital (Urbano y Veciana, 2001).

En sus inicios, hacia comienzos de la década de 1980, las incubadoras tan solo ofrecían un espacio físico donde las empresas podían instalarse, compartir un equipamiento básico de tipo administrativo, obtener una información acerca de permisos, patentes y préstamos; recibir apoyo y asesoramiento en técnicas de dirección y de marketing; disfrutar de servicios básicos a bajo costo; encontrar acceso a capital (Peterson, 1985; Allen y Syedur, 1985). Sin embargo, hoy día las incubadoras han evolucionado hasta convertirse en instrumentos que proporcionan servicios, asesoramiento y un ambiente adecuado para la creación y el crecimiento de nuevas empresas.

Disponen de un amplio y variado conjunto de programas de apoyo al emprendedor, que con diferente grado de especialización, ofrecen: 1) apoyo técnico gerencial a las empresas en su proceso de consolidación, ayudándolas a superar de forma rápida las barreras técnicas, gerenciales y de mercado; 2) capacitación emprendedora un ambiente apropiado y una gerencia dinámica; 3) optimización de costos para las empresas de nueva creación durante el período de incubación; 4) búsqueda de nuevos socios estratégicos; 5) búsqueda de oportunidades en la interacción entre el sector empresarial y las instituciones académicas, de investigación y de innovación.

Tal como señalan Bruneel et al. (2012), en la actualidad se está en presencia de un nuevo concepto de Incubadora. Aún con la misión de promover la creación de empresas para favorecer el desarrollo local, las incubadoras comienzan a orientarse hacia el mercado (Baldini, 2010; Mosey et al., 2012). Esta evolución, más propia de una organización comercial, en gran medida responde a la creciente competencia establecida entre incubadoras y parques científicos y tec- 
Valorando los programas de apoyo al emprendedor en las incubadoras de empresas... Cerdán-Chiscano, Mónica; Jiménez-Zarco, Ana Isabel y Torrent-Sellens, Joan

nológicos gestionados por instituciones de diferente naturaleza ${ }^{1}$. Los beneficios que como institución se derivan de su gestión, junto al impacto que en términos de desarrollo económico social ofrece su instalación, hacen de la incubadora una unidad de negocio sostenible (Potts, 2010:713), pero sobretodo una organización con un potencial extraordinario para la creación de sinergias entre empresas ${ }^{2}$.

Es por ello que su gestión, orientada hacia el mercado, principalmente hacia su cliente, se traduce en: 1) un portafolio que gana en amplitud, así como en profundidad; 2) una oferta de programas y servicios centrados en el emprendedor lo suficientemente atractiva y valiosa para atraer a nuevas empresas, 3) una estrecha vinculación a instituciones de prestigio y redes de emprendimiento, que permitan consolidar una reputación sólida y una imagen -corporativa- de la incubadora ligada a la calidad, la excelencia y el éxito.

Bajo esta nueva orientación, el emprendedor, como cliente potencial de la incubadora, se convierte en su centro de atención (Baldini, 2010). De ahí el interés por parte de estas instituciones en conocer sus características, y el modo en que éstas pueden incidir en la valoración rea- lizada sobre los programas de apoyo que ofrecen.

\section{Valoración de los programas de apoyo: variables determinantes}

El portafolio de servicios ofrecido por la incubadora es el mismo para todas las nuevas empresas que a él se aproximan. Asimismo, entre unas incubadoras $y$ otras existen pocas diferencias en cuanto a su oferta de programas de apoyo al emprendedor. Entonces, ¿qué es lo que marca la diferencia?, ¿por qué en una incubadora un servicio puede recibir diferentes valoraciones?, ¿qué hace que un programa ofrecido al emprendedor por un incubadora sea mejor valorado que otro? Las características del emprendedor y de la empresa parece tienen mucho que ver en la explicación de estas distintas valoraciones.

De forma particular, la revisión de diferentes trabajos en el campo del Management y el emprendimiento han permitido identificar ciertos factores que afectan al resultado de este proceso (Eisenhardt y Schoonhoven, 1990; Parket y van Praag, 2012).

1 Desde comienzo de la década de 1990 gran número de instituciones han creado parques científicos y tecnológicos ofreciendo el servicio de incubadora. Entre las mismas se encuentran administraciones públicas locales y autonómicas, universidades públicas, privadas, fundaciones y consorcios públicos, entre otros.

2 Es posible que la incubadora por si misma constituya dentro del parque una unidad de negocio poco rentable. Pero es cierto que la incubadora constituye una excelente cantera, tanto para la creación de empresas dentro de la universidad (spin-off universitarias, Empresas de Base Tecnológicay Empresas basadas en conocimiento), como para la atracción de otra externas (spin outs y Start ups). 


\subsection{El perfil del emprendedor}

El emprendedor es el verdadero artífice de la creación de una nueva empresa, ya que él identifica la oportunidad, concibe la idea de negocio y la transforma en una realidad (Dvir et al., 2010), es por ello, que para el correcto diseño de programas de apoyo al emprendedor sea necesario conocer características sociodemográficas y psicológicas. Estas características, no sólo determinaran la capacidad del emprendedor para liderar su proyecto de forma exitosa, sino que también incidirán en la valoración que realice de los programas de apoyo desarrollados por la incubadora.

Druilhe y Garnsey (2004), y Parket y van Praag (2012), identifican la capacidad de los emprendedores para gestionar el crecimiento empresarial con dos características de su perfil demográfico: la formación académica y su experiencia profesional. Mientras que, en relación a las características psicológicas del emprendedor, Dvir et al. (2010) identifican: dinamismo y proactividad; intuición; capacidad de organización; mentalidad innovadora, preferencia por el riesgo, y capacidad de diálogo del emprendedor como factores determinantes del éxito y del crecimiento de una nueva iniciativa empresarial.

Los individuos con una formación académica más elevada, disponen de un mayor nivel de conocimiento formal acumulado, lo que ofrece mayores rendimientos para la organización. Parker y Van Praag (2012), apuntan la existencia de una relación directa y significativa entre el nivel educativo del emprendedor y su capacidad de adaptación a las diferentes actividades que requiere la organización, en especial la capacidad de res- puesta a las situaciones de riesgo asociadas al crecimiento empresarial. No obstante, para la puesta en marcha de una nueva iniciativa de negocio, el emprendedor no sólo debe disponer de conocimientos específicos relativos al producto, servicio o tecnología que se desarrollará y comercializará. También es necesario que disponga de conocimientos y habilidades propias en el ámbito del Management y del marketing. Withers et al. (2011) han evidenciado que estos conocimientos son de gran utilidad para la identificación de oportunidades de negocio y, posteriormente, para la toma de decisiones relativas a la combinación de recursos y capacidades para desarrollar y explotar la nueva iniciativa de negocio.

Por otra parte, la experiencia acumulada por el emprendedor también influye de forma positiva sobre el potencial de crecimiento de las empresas. Jo y Lee (1996) reconocen la importancia de la experiencia adquirida a través de la participación en la creación de nuevas empresas y el incremento en el grado de la supervivencia de las mismas. Ucbsasaran et al. (2009), y Parker y van Praag (2012), explican esta vinculación a través de la mayor información que poseen los empresarios sobre los problemas y obstáculos que pueden encontrarse en los primeros años de la vida de la empresa, así como a través del "saber hacer" adquirido para resolverlos. En la misma línea, Ucbasaran et al. (2009), y Parker y van Praag (2012), señalan que el conocimiento adquirido a través de la experiencia profesional es de gran utilidad a la hora de detectar posibles oportunidades de negocio en el proceso de creación de nuevas empresas. 
Valorando los programas de apoyo al emprendedor en las incubadoras de empresas... Cerdán-Chiscano, Mónica; Jiménez-Zarco, Ana Isabel y Torrent-Sellens, Joan

También se ha observado que determinadas características de la personalidad del emprendedor son fundamentales en la creación de la empresa y su posterior crecimiento. Dvir et al. (2010) señalan, entre las más importantes, la preferencia por el cambio, la creatividad, la intuición, y la ambición. En la misma línea, desde el ámbito de la psicología organizacional, Rauch y Frase (2005) señalan entre los factores más importantes: dinamismo y proactividad; intuición; capacidad de organización; mentalidad innovadora; preferencia por el riesgo; y capacidad de diálogo con otros agentes. Particularmente, estos factores son clave por cuanto tienden a traducirse en: 1 ) un interés en actividades específicas en las que el emprendedor puede descubrir y desarrollar nuevas oportunidades de negocio; y 2) una actitud positiva para aprender, y una predisposición a estar atento a toda información relativa al entorno (mercado).

Estos elementos no sólo son determinantes en la identificación de una oportunidad de negocio, sino que también son prioritarios para explicar la capacidad del emprendedor para desarrollar y explorar una nueva idea de negocio. El nivel educativo del emprendedor, su experiencia y personalidad influirán en las fortalezas y debilidades de la nueva organización. Del mismo modo, también serán determinantes a la hora de establecer los recursos que la nueva empresa necesita -conocimientos, contactos, infraestructuras, entre otros-, y la valoración que el emprendedor realizará de los programas y servicios que recibe de la incubadora. Ante esta evidencia, se formula la siguiente pregunta de investigación:
P1. ¿Influye el perfil de emprendedor en su valoración sobre los programas de apoyo ofrecidos por la incubadora?

\subsection{Edad de la empresa}

La edad de la empresa es una variable que incide en la valoración que el emprendedor realiza de los programas de apoyo ofrecidos por la incubadora (Schwartz, 2012). Cuanto más madura es la empresa, más tiempo lleva instalada en la incubadora, mayor es el conocimiento que tiene sobre los servicios ofrecidos $\mathrm{y}$, por consiguiente, posee más argumentos y juicios para valorarlos. Por otra parte, el tiempo de estancia, es proporcional a la experiencia que la empresa tiene en el desarrollo de su negocio, y por tanto, diferentes sus necesidades en cuanto a recursos y conocimientos (Peña-Vinches et al., 2011).

En el momento inicial de incubación, el emprendedor necesita los servicios más básicos del portafolio de productos y servicios: locales; de administración y secretaria; soporte en la realización del plan de empresa; apoyo en la tramitación y gestión de financiación y ayudas públicas, entre otros. A medida que la empresa crece, los productos y servicios requeridos pasan a ser más sofisticados, por ejemplo en materia de necesidades tecnológicas. Por lo tanto, los programas demandados en la fase de crecimiento y consolidación empresarial pasan a ser más especializados, puesto que deben aportar valor al cliente: asesorías especializadas en financiación; en aspectos legales vinculados con negociaciones con empresas de capital riesgo; internacionalización, e innovación son entre 
otros algunos de los servicios más avanzados ofrecidos en la cartera de las incubadoras.

En este contexto, se plantea la siguiente pregunta de investigación:

$P 2$. ¿Influye la edad de la empresa en su valoración sobre los programas de apoyo recibidos de la incubadora?

\subsection{Facturación, tasa de crecimiento y dimensión de de la empresa}

La facturación constituye un buen indicador de la salud de la empresa, así como también de su mayor o menor grado de dinamismo y fortaleza. En este sentido, es de suponer que aquellas empresas que alcanzan cifras de facturación elevadas valoraran con más intensidad que la incubadora ofrezca programas de apoyo más especializados (Alonso y Fageda, 2007). Especialmente aquellos servicios relacionados con el asesoramiento en el terreno de la innovación o en la introducción en nuevos mercados, así como la puesta en contacto con otros posibles socios y/o clientes. Considerando este argumento, se establece la siguiente pregunta de investigación:

P3. ¿Influye el nivel de facturación de la empresa en su valoración sobre los programas de apoyo ofrecidos por la incubadora?

Obtener una cifra de facturación elevada es uno de los principales objetivos que una empresa se marca a corto plazo. Pero éste objetivo tan sólo es un paso para alcanzar otro más importe en el medio y largo plazo: crecer. Para algunas empresas el objetivo puede cifrarse en mantener una tasa de crecimiento constante, sin embargo para otras puede ser fundamental obtener altas tasas de crecimiento a lo largo del tiempo. Sea cual sea el objetivo, la finalidad última de una empresa que crece es seguir creciendo. Para ello, buscará recursos y conocimientos especializados que favorezcan esta tendencia.

Habitualmente el crecimiento de la empresa se mide en base a incrementos en el volumen de ventas, la cifra de facturación o el volumen de exportaciones. En este sentido, cabe esperar que las empresas valoraran de forma positiva aquellos programas y servicios ofrecidos por la incubadora que favorezcan el desarrollo de nuevos productos y procesos, o la introducción de la empresa en nuevos mercados, principalmente internacionales. Tomando en consideración este argumento, se plantea la siguiente pregunta de investigación:

P4. ¿Influye la tasa de crecimiento de la empresa en su valoración sobre los programas de apoyo ofrecidos por la incubadora?

Algunas empresas no tienen como objetivo principal incrementar sus volumen de ventas, o su presencia en los mercados internaciones, sino que su interés se centra en alcanzar un tamaño adecuado a su nivel de actividad o a su volumen de negocio. Las razones para alcanzar el tamaño óptimo pueden variar desde la necesidad de operar en nichos de mercado atractivos pero de pequeña dimensión, hasta estar gestionadas por un empresario que desea mantener la estructura familiar de la empresa.

El tamaño también influirá en el tipo de recursos y programas que la empresa demandará a la incubadora, así como en su valoración del portafolio recibido 
Valorando los programas de apoyo al emprendedor en las incubadoras de empresas... Cerdán-Chiscano, Mónica; Jiménez-Zarco, Ana Isabel y Torrent-Sellens, Joan

(Eisenhardt y Schoonhoven, 1990). Una microempresa o una pyme principalmente necesitarán recursos financieros para desarrollar su actividad, o formación básica en determinadas áreas de su proceso de generación de valor. Es por ello que programas destinados a facilitar el acceso a la financiación pública o a la formación básica serán bien valorados. Por el contrario, las empresas de mayor tamaño, con mayores recursos y personal más formado, valorarán positivamente programas y servicios con mayores grados de especialización. En este sentido, se establece la siguiente pregunta de investigación:

P5. ¿Influye la dimensión de la empresa en su valoración sobre los programas de apoyo ofrecidos porla incubadora?

\subsection{Sector de actividad}

El sector de actividad donde se posiciona la nueva empresa condiciona las necesidades de recursos y conocimientos que se necesitan para el desarrollo de la nueva iniciativa de negocio. Por ejemplo, en ciertos sectores como el sector de las Tecnologías de la Información y la Comunicación (TIC), la nanotecnología o la biotecnología, el producto o servicio ofertado se caracteriza por un alto nivel de especialización, un fuerte componente tecnológico y un ciclo de vida sumamente corto.

En este caso, la demanda de la empresa en período de incubación estará orientada a la obtención de conocimientos técnicos, la financiación, el acceso a equipos altamente especializados, o el asesoramiento en procesos de innovación. Por el contrario, en otros sectores de actividad con un nivel de uso de la tec- nología y el conocimiento menos intensivo, las empresas valorarán de su incubadora recibir asesoramiento y formación en el terreno del e-commerce, o apoyo en actividades de comunicación y promoción, entre otras.

Definitivamente, en su oferta de programas y servicios las incubadoras deben considerar las distintas necesidades de sus empresas y, en especial, las derivadas del sector de actividad, generalmente vinculadas con la intensidad de uso de la tecnología y el conocimiento. En este contexto, se formula la siguiente pregunta de investigación:

P6. ¿Influye el sector de actividad al que la empresa pertenece en su valoración sobre los programas de apoyo ofrecidos por la incubadora?

\subsection{Grado de innovación de la empresa}

Innovar es mucho más que crear nuevos productos o servicios, desarrollar nuevos procesos, o modificar sus estructuras. Consiste en buscar de forma sistemática oportunidades para hacer cosas nuevas o de una forma nueva, de modo que se aporte valor a los clientes y a la propia empresa. De ahí que una empresa innovadora acepta el cambio, y lo hace partiendo de su propia cultura. Cambiar como modo de transformarse, evolucionar o adaptarse es una cuestión interna de la empresa, y el motor del cambio debe vincularse a una estrategia de negocio (Schneider y Veugelers, 2010).

Como respuesta a los cambios del mercado, la empresa diseña, desarrolla y ofrece nuevos productos y servicios. Asimismo, y teniendo en cuenta criterios de 
eficacia y eficiencia, es posible rediseñar o implementar nuevos procesos, bien, como respuesta a cambios en el entorno, o bien como respuesta a factores internos. La empresa puede adoptar una estructura flexible, apostar por la cooperación, mejorar la comunicación, usar más intensivamente las tecnologías de información y comunicación (TIC's), o desarrollar el trabajo en equipo y la polivalencia en sus trabajadores (Wichitchany et al., 2012). Por este conjunto de motivos el grado de innovación de la empresa influye de manera importante en el tipo de programas que éstas demandan a las incubadoras, así como también en su valoración. A medida que el grado de innovación sea mayor, la empresa demandará a la incubadora servicios más especializados, e incluso novedosos. En este sentido, se formula la siguiente pregunta de investigación:

P7 ¿Influye el grado de innovación de la empresa en su valoración sobre los programas de apoyo ofrecidos por la incubadora?

\section{Estudio de las incubadoras de empresas en Cataluña}

Con el ánimo de dar respuesta a las preguntas de investigación anteriormente planteadas, se procedió a desarrollar una investigación descriptiva sobre una muestra de empresas creadas en el entorno de las incubadoras en Cataluña.

La elección del territorio responde tanto al perfil de la empresa catalana, como a la fuerte dinámica empresarial experimentada en Cataluña durante la última década. El tejido empresarial en Cataluña está mayoritariamente integrado por empresas de muy pequeña dimensión (más del 92\% de empresas en este territorio tienen menos de 10 trabajadores), y su supervivencia -determinada por su capacidad innovadora- queda supeditada tanto a las particularidades de sus procesos internos de gestión, como a las características y dinámicas del entorno que las rodea.

La pequeña dimensión de la empresa catalana establece consecuencias directas sobre su potencial para generar endógenamente innovaciones y sobre su capacidad de asimilación de nuevas tecnologías y conocimientos del exterior. De hecho, $y$ ante la debilidad innovadora de la micro y pequeña empresa en Cataluña, la capacidad del tejido institucional, en especial las incubadoras de empresas, para promocionar la innovación es imprescindible para garantizar la supervivencia y el crecimiento de este tejido mayoritario de nuevas empresas.

\subsection{Incubadoras de empresas en Cataluña}

Cataluña ha tenido tradicionalmente una tasa elevada de emprendedores, lo que ha determinado su estructura industrial, formada mayoritariamente por empresas de dimensión muy reducida (Alonso y Fageda, 2005:32). En el territorio catalán, el fenómeno de las incubadoras de empresas se inició a principios de los años 1980 , siguiéndose para su creación dos estrategias diferenciadas (Smilor y Gill, 1986):

- La primera, basada en la renovación de edificios antiguos e infrautilizados, se centra más en ofrecer a agentes emprendedores, acceso al espacio, más que en el desarrollo de los programas de sopor- 
Valorando los programas de apoyo al emprendedor en las incubadoras de empresas... Cerdán-Chiscano, Mónica; Jiménez-Zarco, Ana Isabel y Torrent-Sellens, Joan

te al emprendedor. En este caso, el valor aportado por la incubadora es la reducción de los costos iniciales de la empresa.

- La segunda estrategia era una tentativa más consciente para fomentar y consolidar nuevas empresas en el territorio. Con ella, algunas incubadoras desarrollan una oferta de programas y servicios con el objetivo de ofrecer un soporte integral a los nuevos emprendedores.

Las incubadoras de empresas en Cataluña ofrecen tanto espacios físicos adaptados a las necesidades del emprendedor, así como toda una gama de servicios de apoyo empresarial. A finales de 2006, en Cataluña existían un total de 29 incubadoras, las cuales se encontraban mayoritariamente localizadas en la provincia de Barcelona ${ }^{3}$. En la mayoría de ocasiones, formaban parte de una sociedad municipal con capital aportado por las instituciones locales -ayuntamientos- y estaban especializadas en empresas de servicios.

Para la aproximación del estudio se utilizó una muestra de 29 microempresas y PYMES creadas en el periodo 20002006 y que desarrollaban su actividad en incubadoras de empresas en Cataluña. El reducido tamaño de la muestra se justifica dado que el objeto de estudio es una población finita, y circunscrita a un territorio concreto. No obstante, este hecho también limita la capacidad de generalización y extrapolación de las conclusiones obtenidas (Cuadro 1).
Del total de las 29 incubadoras localizadas en Cataluña, tan sólo se pudo acceder a 13. Ello fue debido a que algunas de las entidades no se mostraron interesadas en participar en las encuestas o bien en el momento de contactar con ellas no correspondían a la tipología de incubadora de empresas municipal objeto de estudio.

La recogida de información se realizó mediante una encuesta personal administrada a los emprendedores a través de los responsables de las incubadoras de empresas, debía ser respondida de forma electrónica en el lugar donde desarrollan su actividad empresarial. Las empresas de nueva creación que forman parte de la muestra cumplen las siguientes condiciones: 1) tener menos de tres años de vida en período de incubación; 2) localizar su actividad empresarial dentro de una incubadora de empresas municipal de Cataluña; 3) haber participado en alguno de los programas de apoyo ofrecidos por la incubadora.

La Tabla 1, recoge la información obtenida en el trabajo de campo. La indicación $X$ señala que la medida de soporte es ofrecida al emprendedor. Siguiendo a Urbano y Vecina (2001), las principales medidas de apoyo a la creación de empresas se clasifican en medidas económicas y no económicas Las medidas no económicas son: la información, el asesoramiento, la formación y los programas de incubadoras de empresas. Mientras,

3 En Cataluña en el año 2006 hay 29 incubadoras de empresas municipales que desarrollan su actividad en el territorio de Cataluña: 18 pertenecen a la provincia de Barcelona, 5 a la provincia de Tarragona, 3 a la provincia de Girona y 2 a la provincia de Lérida. 


\section{Cuadro 1 \\ Ficha técnica del estudio}

Población

Unidad de muestra

Ámbito geográfico

Censo poblacional

Diseño de la muestra

Tamaño de la muestra

Nivel de confianza

Método de recogida de información
Microempresas y PYMES creadas en el periodo 2000-2006 y que desarrollan su actividad en incubadoras de empresas en Cataluña

Empresas actuales instaladas en alguna de las incubadoras de empresas de Cataluña

Cataluña

400 empresas

Muestreo no aleatorio por conveniencia, a partir de los criterios subjetivos especificados

29

$95,5 \%$

Entrevista por correo electrónico mediante cuestionario estructurado

Fuente: Elaboración propia.

ente las principales medidas económicas ofrecidas, se destacan: bonificaciones fiscales, capital riesgo y capital riesgo en condiciones favorables ${ }^{4}$, créditos a tipos de interés subvencionados a partir de convenios firmados por las administraciones públicas y entidades financieras, subvenciones a fondo perdido, cada vez menos frecuentes, entre otros.

Junto a las limitaciones de generalización de las conclusiones obtenidas, el reducido tamaño de muestra también restringe las escalas de medida utilizadas en las diferentes variables de estudio, así como en el tipo de técnica estadística a utilizar, forzando la utilización de análisis estadísticos no paramétricos.

La dimensión de la muestra aconseja utilizar métricas que no ofrezcan una amplia dispersión de la información. De ahí que buena parte de las variables, medidas en el cuestionario a través de escalas Likert, fueron recodificadas a dicotómicas. Otras variables, de naturaleza latente, fueron creadas a partir de métodos estadísticos multivariantes de clasificación y agrupación, como es el caso del Análisis Cluster jerárquico. El Cuadro 2 , recoge las variables utilizadas en el estudio.

4 La opción financiera de capital riesgo va dirigida a empresas de base tecnológica (EBT). Algunas de las entidades que promueven este tipo de medida económica en Cataluña son, entre otros, la empresa Invertec del Cidem (http://www.cidem.com), la empresa Barcelona Emprèn (http://www.bcnempren.es), y la sociedad Innova31 de la Universidad Politécnica de Cataluña (UPC) (http://www.innova31.com). 
Valorando los programas de apoyo al emprendedor en las incubadoras de empresas... Cerdán-Chiscano, Mónica; Jiménez-Zarco, Ana Isabel y Torrent-Sellens, Joan

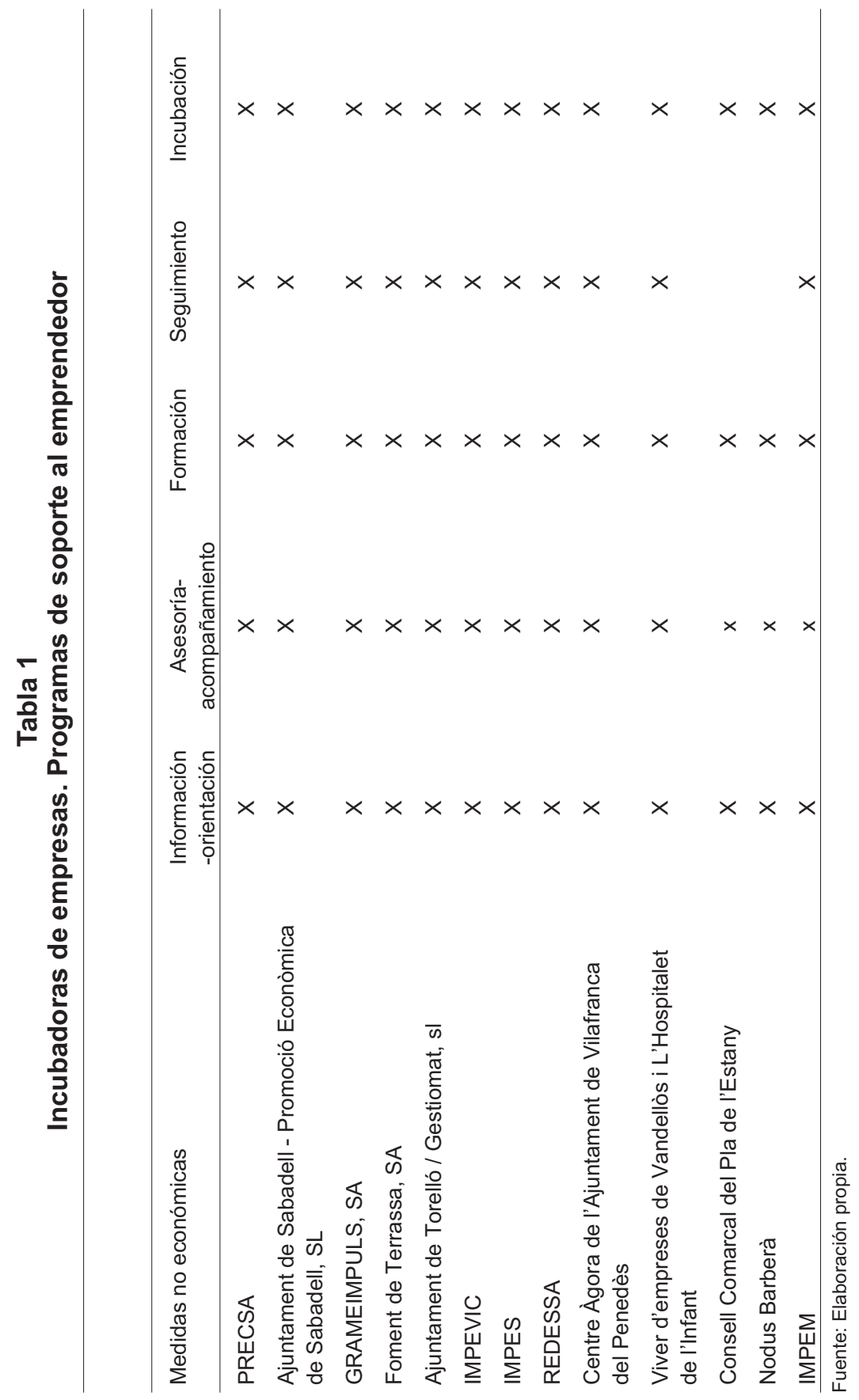




\section{Cuadro 2 \\ Variables utilizadas en el estudio}

\begin{tabular}{|c|c|}
\hline Variable & Descripción \\
\hline Sector de actividad & $\begin{array}{l}\text { Sector donde la empresa desarrolla su actividad. La variable original } \\
\text { mostraba un total de } 17 \text { categorías diferentes de sectores de actividad. } \\
\text { A fin de reducir su dispersión, la variable ha sido transformada, diferen- } \\
\text { ciando entre actividades intensivas y no intensivas en el uso del cono- } \\
\text { cimiento. }\end{array}$ \\
\hline Año de creación & $\begin{array}{l}\text { Año en que se creó la empresa. Esta variable contempla } 6 \text { valores, en } \\
\text { función de que el año de creación fuera 2001, 2002, 2003, 2004, 2005, } \\
\text { y } 2006 \text {. }\end{array}$ \\
\hline $\begin{array}{l}\text { Dimensión } \\
\text { de la empresa }\end{array}$ & $\begin{array}{l}\text { Número de trabajadores que tiene la empresa en momento de realiza- } \\
\text { ción de la encuesta. Se trata de una variable categórica donde el valor } \\
1 \text { indica que no tiene ningún trabajador; } 2 \text {, cuando tiene entre } 1 \text { y } 3 \text { tra- } \\
\text { bajadores; } 3 \text { tiene entre } 4 \text { y } 10 \text { trabajadores; } 4 \text { si tiene más de } 10 \text { traba- } \\
\text { jadores. }\end{array}$ \\
\hline Facturación & $\begin{array}{l}\text { Cifra de facturación durante el año } 2005 \text { medido en euros. Es una va- } \\
\text { riable categórica que adopta el valor } 1 \text { cuando la empresa ha facturado } \\
\text { menos de } 30.000 \text { euros; } 2 \text {, cuando ha facturado entre } 30.000 \text { y } 60.000 \\
\text { euros; } 3 \text { cuando ha facturado entre } 60.000 \text { y } 120.000 \text { euros; y } 4 \text { si ha } \\
\text { facturado más de } 120.000 \text { euros. }\end{array}$ \\
\hline Tasa de crecimiento & $\begin{array}{l}\text { Tasa de crecimiento alcanzada por la empresa en el } 2005 \text {, con respec- } \\
\text { to a su cifra de ventas del año } 2004 \text {. La variable es categórica y presen- } \\
\text { ta el valor } 0 \text { cuando la empresa ha obtenido una tasa decreciente; } 1 \text {, } \\
\text { cuando no ha habido variación con respecto al año anterior; } 3 \text { cuando } \\
\text { se ha producido un crecimiento moderado, y } 4 \text { cuando la tasa de creci- } \\
\text { miento es alta. }\end{array}$ \\
\hline Empresa innovadora & $\begin{array}{l}\text { Indica si la empresa ha realizado innovaciones de carácter tecnológi- } \\
\text { co. Es una variable dicotómica que presenta el valor } 0 \text { si la empresa no } \\
\text { ha innovado, y } 1 \text { si ha innovado. }\end{array}$ \\
\hline Perfil del emprendedor & $\begin{array}{l}\text { Variable que muestra el perfil sociodemográfico y psicológico del em- } \\
\text { prendedor. La variable adopta el valor } 1 \text { si se trata de un emprendedor } \\
\text { pasivo y } 2 \text { si se trata de un emprendedor proactivo. }\end{array}$ \\
\hline $\begin{array}{l}\text { Valoración dada a los ser- } \\
\text { vicios básicos ofrecidos } \\
\text { por la incubadora }\end{array}$ & $\begin{array}{l}\text { Valoración dada por el emprendedor a los diferentes servicios básicos } \\
\text { ofrecidos por la incubadora. La variable original estaba medida en una } \\
\text { escala Likert } 5 \text { puntos, pero fue transformada a una escala dicotómica } \\
\text { donde adopta el valor } 1 \text { si la valoración se encuentra por debajo de la } \\
\text { media de las valoraciones de todos los servicios básicos, y } 2 \text { si se en- } \\
\text { cuentra por encima. }\end{array}$ \\
\hline
\end{tabular}

Fuente: Elaboración propia. 
Valorando los programas de apoyo al emprendedor en las incubadoras de empresas... Cerdán-Chiscano, Mónica; Jiménez-Zarco, Ana Isabel y Torrent-Sellens, Joan

En el proceso de construcción de variables para el análisis destaca, por su complejidad, la construcción de dos indicadores. En primer lugar, el indicador del emprendedor fue creado a partir del resultado obtenido tras la realización de un análisis cluster jerárquico. Las características sociodemográficas consideradas fueron la formación recibida (el nivel de estudios, su formación en el campo de la gestión de empresas y las finanzas, si ha desarrollado formación o reciclaje durante los dos últimos años) y la experiencia previa en la creación de empresas. Mientras que entre las características psicológicas se consideraron: su carácter dinámico y proactivo, su capacidad organizativa, su mentalidad innovadora, su capacidad para asumir riesgo e invertir dinero, su capacidad para dialogar con los trabajadores, su interés por ganar dinero a corto plazo, o su interés por crecer a ritmo lento para contratar trabajadores.
La Tabla 2 distingue entre dos grupos de individuos bien diferenciados. El primero, conformado por 19 individuos representa al emprendedor proactivo como aquel individuo con capacidad organizativa, formación a nivel de master y conocimientos en finanzas y gestión. Dispone de una mentalidad innovadora, asume riesgos e invierte su dinero, dialoga con trabajadores y tiene experiencia previa en la creación de otras empresas. Mientras, el segundo grupo formado por 10 individuos, representa al emprendedor pasivo el cual no dispone de capacidad organizativa ni de dialogo, no dispone de formación superior, no experiencia previa ni tampoco conocimientos de gestión de empresas. No tiene mentalidad innovadora, ni tiene interés por invertir recursos en la empresa dado su aversión al riesgo.

En lo que respecta a la valoración dada por el emprendedor al conjunto de

\section{Tabla 2}

Historial de iteraciones realizadas, e información sobre los clúster obtenidos

\begin{tabular}{ccccc}
\hline & \multicolumn{2}{c}{$\begin{array}{l}\text { Cambio en los centros } \\
\text { de los conglomerados }\end{array}$} & Conglomerado 1 & 10 \\
\hline Iteración & 1 & 2 & Conglomerado 2 & 19 \\
1 & 1,591 & 1,900 & Validos & 29 \\
2 &, 224 &, 118 & & \\
$3^{5}$ &, 000 &, 000 & & \\
\hline
\end{tabular}

Fuente: Elaboración propia.

5 Se ha logrado la convergencia debido a que los centros de los conglomerados no presentan ningún cambio o éste es pequeño. El cambio máximo de coordenadas absolutas para cualquier centro es de ,000. La iteración actual es 3. La distancia mínima entre los centros iniciales es de 4,123 . 
servicios básicos y avanzados de la incubadora cabe señalar que ambas variables se crearon a partir de la valoración dada al conjunto de servicios básicos y avanzados, que tal y como muestra el Cuadro 3, son ofrecidos por las incubadoras en Cataluña.
Cada uno de estos servicios fue valorado por separado por el emprendedor, mediante una escala Likert de 5 puntos donde el valor 1 indicaba una baja importancia, y el valor 5 una gran importancia. A partir de las valoraciones dadas por cada emprendedor a cada servicio, se procedió

\section{Cuadro 3 \\ Servicios Básicos y Avanzados ofrecidos por las incubadoras en Cataluña}

\begin{tabular}{|c|c|}
\hline Servicios Básicos & Servicios Avanzados \\
\hline Realización del plan de empresa & $\begin{array}{l}\text { Asesoramiento especializado en Innova- } \\
\text { ción de empresa }\end{array}$ \\
\hline Actualización del plan de empresa & $\begin{array}{l}\text { Asesoramiento especializado en marke- } \\
\text { ting o estudios de mercado }\end{array}$ \\
\hline Asesoramiento de creación de empresa & $\begin{array}{l}\text { Asesoramiento especializado técnico o } \\
\text { científico }\end{array}$ \\
\hline $\begin{array}{l}\text { Asesoramiento especializado en búsqueda de fi- } \\
\text { nanciación/ayudas }\end{array}$ & $\begin{array}{l}\text { Asesoramiento especializado en fiscali- } \\
\text { dad y jurídica }\end{array}$ \\
\hline $\begin{array}{l}\text { Tramitación de ayudas financieras y seguimiento de } \\
\text { la concesión }\end{array}$ & $\begin{array}{l}\text { Asesoramiento especializado en contabi- } \\
\text { lidad, finanzas }\end{array}$ \\
\hline Formación en innovación de empresa & $\begin{array}{l}\text { Asesoramiento especializado en marke- } \\
\text { ting o estudios de mercado }\end{array}$ \\
\hline Formación en áreas de empresa & $\begin{array}{l}\text { Asesoramiento especializado en estrate- } \\
\text { gia empresarial }\end{array}$ \\
\hline Asistencia a ferias/congresos & $\begin{array}{l}\text { Búsqueda de contactos/colaboradores } \\
\text { (networking) }\end{array}$ \\
\hline $\begin{array}{l}\text { Acciones de difusión de la empresa (datos de la em- } \\
\text { presa en la red...) }\end{array}$ & $\begin{array}{l}\text { Búsqueda de proveedores o socios tecno- } \\
\text { lógicos }\end{array}$ \\
\hline Servicios de asesoramiento y formación on-line & $\begin{array}{l}\text { Proyectos europeos o nacionales (Pro- } \\
\text { grama Marco, becarios...) }\end{array}$ \\
\hline \multicolumn{2}{|l|}{ Búsqueda de locales de salida de la incubadora } \\
\hline \multicolumn{2}{|l|}{ Jornadas realizadas por la incubadora de empresas } \\
\hline \multicolumn{2}{|l|}{ Difusión de concursos públicos o licitaciones. } \\
\hline \multicolumn{2}{|l|}{ Servicio de recibo de noticias de interés y actualidad } \\
\hline \multicolumn{2}{|l|}{ Servicio de administración, secretaria y generales } \\
\hline $\begin{array}{l}\text { Servicio de alquiler de espacios, locales y despa- } \\
\text { chos e infraestructuras en la incubadora }\end{array}$ & \\
\hline
\end{tabular}

Fuente: Elaboración propia. 
Valorando los programas de apoyo al emprendedor en las incubadoras de empresas... Cerdán-Chiscano, Mónica; Jiménez-Zarco, Ana Isabel y Torrent-Sellens, Joan

a calcular la valoración media (a través de la media ponderada) de los servicios básicos y la de los servicios avanzados. Las variables obtenidas a través de este paso intermedio, medidas en una escala métrica, fueron dicotomizadas, considerando que el valor presentado estuviera por encima o por debajo de la media de la variable. Este proceso de análisis constituye la construcción del indicador de valoración del emprendedor a los servicios ofrecidos por la incubadora (Tabla 3).

\section{Primeras evidencias en las empresas innovadoras catalanas}

Urbano y Veciana (2001) han detectado que casi un $60 \%$ de los empresarios catalanes hacen una valoración negativa sobre los programas de apoyo a la creación de nuevas empresas en Catalu- ña. Estos mismos autores afirman que las instituciones públicas implicadas en la gestión de las incubadoras dependen excesivamente del ciclo político en el que están inmersos.

En línea con los resultados anteriores, se encuentra como un elevado porcentaje de emprendedores valoran de forman negativa los programas de apoyo ofrecidos por las incubadoras. No obstante, dicha valoración varía de forma importante atendiendo a la naturaleza del servicio ofrecido por la incubadora. Para aquellos programas de apoyo cualificados como básicos, el porcentaje de emprendedores que realizan una valoración baja es del $44,8 \%$. Mientras que para aquellos programas avanzados de apoyo a la creación de nuevas empresas, el porcentaje de emprendedores que realizan una valoración baja se eleva hasta el $65,5 \%$. Estos resultados difieren de los alcanzados por

\section{Tabla 3}

Estadísticos descriptivos de las valoraciones realizadas sobre los programas de apoyo básicos y avanzados ofrecidos por las incubadoras

\begin{tabular}{|c|c|c|c|}
\hline & & $\begin{array}{c}\text { Valoración Programas } \\
\text { Básicos }\end{array}$ & $\begin{array}{c}\text { Valoración Programas } \\
\text { avanzados }\end{array}$ \\
\hline \multicolumn{2}{|l|}{ Media } & 4,75862 & 3,3414 \\
\hline \multicolumn{2}{|l|}{ Mediana } & 4,70000 & 3,1000 \\
\hline \multicolumn{2}{|l|}{ Moda } & 4,300 & 3,00 \\
\hline \multicolumn{2}{|l|}{ Desv. típ. } & 67895 & 69463 \\
\hline \multicolumn{2}{|l|}{ Varianza } & ,4608 & ,483 \\
\hline \multirow[t]{3}{*}{ Percentiles } & 25 & 4,30000 & 2,9000 \\
\hline & 50 & 4,70000 & 3,1000 \\
\hline & 75 & 5,30000 & 3,8500 \\
\hline \multicolumn{2}{|c|}{$\%$ Por debajo de la media } & $44,8 \%$ & $65,5 \%$ \\
\hline \multicolumn{2}{|c|}{$\%$ Por encima de la media } & $55,2 \%$ & $34,5 \%$ \\
\hline
\end{tabular}

Fuente: Elaboración propia. 
(Muhamad et al., 2007) quienes señalan que los emprendedores tienden a valorar positivamente los servicios relacionados con la propia infraestuctura e instalaciones de la incubadora, mientras que otorgan poca importancia los servicios avanzados de asesoramiento y networking.

A tenor de los resultados anteriores tiene sentido pensar que tanto el perfil del emprendedor, como algunas de las características de la empresa influyen de forma importante en la valoración realizada. Por lo que respecta al emprendedor, el resultado encontrado, está en línea con los obtenidos por el Global Entrepreneurship Monitor (2012). Así, el perfil de emprendedor se caracterizaba por ser mayoritariamente un hombre, joven, con estudios superiores. Dos tercios de los individuos que han decidido crear una empresa en el entorno de las incubadoras son hombres (un 52,3\% entre 25 y 35 años), que en su mayoría habían finalizado estudios universitarios $(82,6 \%)$, y presentaban un perfil técnico. Es decir, se caracterizaban por un conocimiento elevado del producto o servicio, más que por disponer de habilidades de gestión o por su conocimiento del mercado donde competían. Este fenómeno, junto al hecho de que buena parte de los emprendedores no poseía experiencia previa en el terreno empresarial $(78,3 \%)$, explica su elevado interés por la formación continuada y el reciclaje. Cerca de un $87 \%$ de los emprendedores realizó algún curso de reciclaje empresarial durante el período de análisis. De hecho, esta formación coincide en el tiempo con el momento en que el emprendedor decidió crear la empresa.

En lo que respecta a la empresa instalada en la incubadora su perfil responde al de una microempresa joven perteneciente al sector servicios, y que ha sido financiada en más de un $50 \%$ por el emprendedor. En concreto, la edad media de las empresas situadas en el entorno de las incubadoras de Cataluña es de 2,5 años, siendo algo superior el número de empresas que superan los 3 años de vida. Sólo un $8,7 \%$ de las empresas instaladas en las incubadoras supera los 5 años de vida y un $4,3 \%$ aún no tiene el primer año de vida empresarial. Hytti (2007) apunta que la edad de la empresa es determinante en el nivel de satisfacción mostrado hacia los servicios ofrecidos ofrecidos por la incubadora. Así las empresas que muestran mayor nivel de satisfacción son aquellas que tienen menos de dos años de vida.

En cuanto al sector de actividad, entre las empresas analizadas destacan aquellas del sector servicios, y principalmente las tecnológicas, con un $48 \%$ del total de la muestra. Le siguen en importancia las empresas de I+D con un $13,5 \%$, y a gran distancia otros servicios como el comercio al por menor y el turismo. En cuanto a su tamaño, se observa como la mayoría de empresas $(78,3 \%)$ disponen de menos de tres trabajadores a jornada completa y el resto $(21,7 \%)$ entre 4 y 10 trabajadores. Así mismo, la dimensión de la empresa en Cataluña es de un $88,1 \%{ }^{6}$ de 5 o menos trabajadores y en España de 76,9\% de traba- 
Valorando los programas de apoyo al emprendedor en las incubadoras de empresas... Cerdán-Chiscano, Mónica; Jiménez-Zarco, Ana Isabel y Torrent-Sellens, Joan

jadores, por tanto la dimensión de la empresa en el entorno de la incubadora municipal es muy parecida al conjunto del territorio catalán.

A fin de analizar la importancia que las características de la empresa y del emprendedor tienen sobre la valoración recibida por los diferentes programas de apoyo a la creación de empresas, ofrecidos por las incubadoras, conlleva a plantear el desarrollo de un análisis no paramétrico basado en la prueba $U$ de Mann-Whitney. A través de ella se procederá a analizar la existencia de diferencias entre las medias de dos poblaciones independientes, que en este caso están constituidas por los programas básicos y avanzados que la incubadora desarrolla para apoyar al emprendedor.

Las Tablas 4 y 5 contienen los resultados del parámetro y el grado de significación de las diferentes variables independientes analizadas tanto para la muestra de servicios básicos como avanzados. El análisis detallado de las mismas, muestra la existencia de diferencias en cuanto a las variables que tienen un efecto significativo sobre la valoración realizada por el emprendedor.

Para la muestra de servicios básicos (Tabla 4) se observa como el perfil del emprendedor es la única variable determinante en la valoración realizada sobre los programas. Mientras que el resto de variables, parecen no presentar ningún efecto al respecto. Esta idea, previamente señalada en trabajos como los de Parket y van Praag (2012) y Withers et al. (2011), ya fue puesta de manifiesto tras la realización de las tablas de contingencia. Así, el se refuerza cuando se realizan las tablas de contingencia entre las diferentes variables consideradas, y se observa cómo el $80 \%$ de los emprendedores realizan una valoración favorable de estos programas, frente al $20 \%$ de los mismos que hacen una valoración negativa.

Por otro lado, y tomando en consideración los servicios avanzados (Tabla 5) se desprende que junto al perfil del empresario, el sector de actividad, la dimensión de la empresa, su facturación, su tasa de crecimiento o su perfil emprendedor son variables que influyen de manera significativa en la valoración que el emprendedor realiza de los programas avanzados que ofrece la incubadora. En este sentido, los resultados obtenidos vienen a reforzar las conclusiones ya obtenidas por Alonso y Fageda (2007); Dvir et al. (2010); Eisenhardt y Schoonhoven (1990) o Schneider y Veugelers (2010) entre otros. Por el contrario, y en relación a la edad de la organización, los resultados alcanzados difieren respecto a los alcanzados por Hytti (2007) quien sostiene que este factor constituye el principal determinante de la satisfacción alcanzada respecto a los servicios de la incubadora.

Con todo ello, y tal como muestra el Cuadro 4, los resultados anteriores nos permiten confirmar la pregunta de investigación 1 para los programas básicos ofrecidos por la incubadora, y las preguntas $1,3,4,5$, 6 y 7 , para los programas de apoyo avanzados que ofrece la incubadora. 


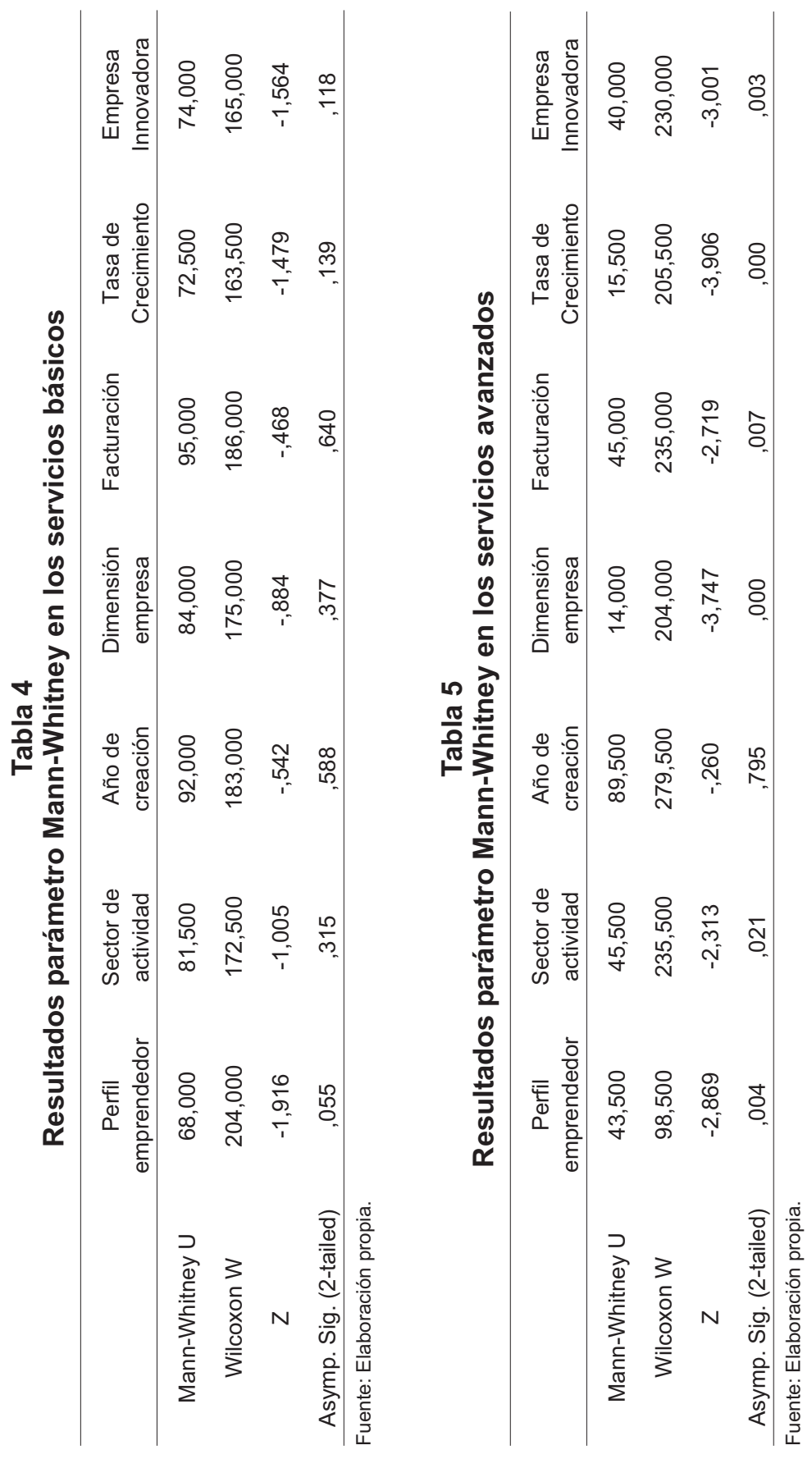


Valorando los programas de apoyo al emprendedor en las incubadoras de empresas... Cerdán-Chiscano, Mónica; Jiménez-Zarco, Ana Isabel y Torrent-Sellens, Joan

\section{Cuadro 4 \\ Preguntas de investigación confirmadas para cada una de las muestras}

\begin{tabular}{lcc}
\hline & Programas básicos & Programas avanzados \\
\hline $\begin{array}{l}\text { P1. El perfil del emprendedor influirá en su valora- } \\
\text { ción sobre los programas de apoyo ofrecidos por } \\
\text { la incubadora }\end{array}$ & aceptada & Aceptada \\
$\begin{array}{l}\text { P2. La edad de la empresa influirá en la valora- } \\
\text { ción sobre los programas de apoyo ofrecidos }\end{array}$ & rechazada & Rechazada \\
$\begin{array}{l}\text { P3. La facturación de la empresa influirá en la va- } \\
\text { loración sobre los programas de apoyo ofrecidos } \\
\text { por la incubadora }\end{array}$ & rechazada & Aceptada \\
$\begin{array}{l}\text { P4. La tasa de crecimiento de la empresa influirá } \\
\text { en la valoración sobre los programas de apoyo } \\
\text { ofrecidos por la incubadora }\end{array}$ & rechazada & \\
$\begin{array}{l}\text { P5. La dimensión de la empresa influirá en la va- } \\
\text { loración sobre los programas de apoyo ofrecidos } \\
\text { por la incubadora }\end{array}$ & rechazada & Aceptada \\
$\begin{array}{l}\text { P6. El sector de actividad al que la empresa per- } \\
\text { tenece influirá en la valoración sobre los progra- } \\
\text { mas de apoyo ofrecidos por la incubadora }\end{array}$ & rechazada & Aceptada \\
$\begin{array}{l}\text { P7 El grado de innovación de la empresa influirá } \\
\text { en la valoración sobre los programas de apoyo } \\
\text { ofrecidos por la incubadora }\end{array}$ & & rechazada \\
\hline
\end{tabular}

Fuente: Elaboración propia.

\section{Conclusiones}

La importancia de las incubadoras es crucial a la hora de potenciar y consolidar la actividad emprendedora. No obstante se observa como existen diferencias importantes en cuanto a los factores que determinan la valoración de los programas de apoyo al emprendedor. De esta manera, a medida que la complejidad o especialización del programa se incrementa, la incubadora ha de tener en cuenta tanto los factores relativos al perfil del emprendedor, como las características de las empresas a las que se dirige. La investigación realizada concluye que el perfil del emprendedor es clave en el di- seño de la oferta de programas. Con independencia de los servicios ofrecidos por la incubadora (básicos o avanzados), la elección, y posterior evaluación, de estos servicios por parte del emprendedor están directamente relacionados con sus necesidades e intereses, en especial con la formación a recibir.

Disponer de estudios superiores puede conllevar a que el emprendedor sea experto en el diseño y fabricación de determinado producto, o proceso. Pero esto, que hasta cierto punto puede constituir la base de una ventaja competitiva en costos o basada en un rápido lanzamiento del producto al mercado, no garantiza el éxito. Junto a los conocimientos técni- 
cos se necesitan otros capacidades, propias de gestión empresarial, ya sean a nivel básico o avanzado.

Incluso aunque el emprendedor disponga de los conocimientos técnicos y de Management necesarios para gestionar su empresa de forma eficiente, siempre existen intereses personales, que le pueden motivar a recibir una formación $o$ un asesoramiento a fin de desarrollar o incrementar sus competencias. De forma innata el individuo puede tener mayor o menor predisposición a desarrollar o a adquirir cierta competencia. En otras ocasiones, la experiencia profesional o la educación recibida pueden ayudarle a ello. Pero, es cierto que algunas competencias requieren de una formación específica muy concreta, y es ahí donde la oferta de servicios y programas de apoyo señalados por la incubadora pueden ser clave. A parte de las características del emprendedor, también deben tomarse en consideración el sector donde la empresa desarrolla su actividad, su dimensión, su facturación (y su evolución), y su predisposición a innovar. Todos ellos son factores determinantes a la hora de demandar y valorar un servicio o un programa de apoyo de la incubadora.

Estas características son especialmente relevantes cuando se trata de la valoración de programas de apoyo avanzados. Los procesos de innovación e internacionalización que caracterizan a las empresas dinámicas y emprendedoras se ven favorecidos si éstas reciben un asesoramiento y apoyo especializado. De manera especial, si la incubadora ofrece servicios destinados a la búsqueda de proveedores o socios tecnológicos, asesoramiento estratégico o el acceso a una red de colaboradores, las empresas incrementan sus probabilidades de éxito futuro. En esta línea, Hytti (2007) sugiere que las empresas orientadas a la internacionalización y al crecimiento global son las que más valor dan a los servicios especializados.

Los resultados alcanzados en este estudio confirman la necesidad de gestionar la incubadora considerando el perfil de los emprendedores y las empresas que alberga. Asimismo, también ponen de manifiesto como la supervivencia de la incubadora pasa por disponer de una oferta de programas y servicios especializados y adaptados a la amplia diversidad de perfiles y tipos de empresas.

Es evidente que el pequeño tamaño de la muestra analizada, así como su circunscripción a un territorio concreto como Cataluña, limitan la capacidad de generalización de las conclusiones alcanzadas. No obstante, también suponen el primer paso para avanzar en el estudio de la gestión eficiente de las incubadoras de empresa, y de su papel en el desarrollo económico local. El avance en el estudio de determinados temas como la influencia de las incubadoras en el desarrollo de empresas en determinados sectores económicos, como el agroalimentario, o el tecnológico o el biosanitario; o el desarrollo de modelos de gestión estratégica que permita el diseño de programas a medida para las empresas en ella instalada, y a la vez incremente la capacidad competitiva de la incubadora, constituyen algunas de las propuestas de investigación futura en este ámbito.

En síntesis, recomienda orientar la gestión de la incubadora hacia el mercado, o lo que es lo mismo, conocer al clien- 
Valorando los programas de apoyo al emprendedor en las incubadoras de empresas... Cerdán-Chiscano, Mónica; Jiménez-Zarco, Ana Isabel y Torrent-Sellens, Joan

te y a la competencia, $y$ tratar de ofrecerle un servicio más valioso y totalmente adaptado a sus necesidades. De esta manera, y previamente al diseño de la cartera de servicios que se ofrecerá, la dirección de la incubadora ha de establecer cuál es el perfil de emprendedor y de empresa al que se desea o se puede llegar. Puede ser que hablar de segmentación del mercado y selección del público objetivo es más propio de una organización comercial, que de un institución pública. Pero puede ser que un tipo de emprendedores o de empresas sean más interesantes $u$ atractivas para la incubadora, $y$ en parte esta decisión puede venir condicionada por la ubicación geográfica de la incubadora, los intereses de la institución pública que le da apoyo, o la limitación de recursos financieros, humanos o tecnológicos.

Considerando el perfil del emprendedor a quienes van dirigidos los programas y servicios ofrecidos por la incubadora, se hace altamente recomendable que esta diseñe una cartera de programas y servicios amplia, profunda y con un nivel elevado de sofisticación o especialización. Nuevamente, los recursos de los que dispone la incubadora serán claves en este punto. Una amplia cartera de servicios puede constituir un atractivo a la hora de atraer a los emprendedores a la incubadora, pero también exigirá el empleo de abundantes recursos de los que la incubadora puede carecer. A pesar de que una especialización del servicio en función del tipo de empresa al que se dirige puede ser una opción inicialmente menos atractiva, en el largo plazo puede suponer la base para la construcción de una ventaja competitiva sostenible. Llegados a este punto, $y$ en vista a las conclusiones alcanzadas, se observa la existencia de un mapa de incubadoras con una diversidad y topología de clientes tan amplia y variada como proyectos empresariales existentes. Así pues, nos planteamos preguntas de cómo las siguientes: ¿en qué incubadora puede el emprendedor recibir mejor servicio? y a su vez, ¿genera el modelo actual de incubadoras una competencia entre sí, duplicando servicios financiados públicamente? y por último, ¿existe un modelo de gestión más eficaz de la incubadora que a su vez incremente el nivel de satisfacción del cliente? La respuesta a las dos primeras preguntas está, en cierto modo, conectada. Cada incubadora funciona independientemente del resto y, por tanto, define en base a sus capacidades y recursos disponibles, los servicios de incubación. Es inevitable, pues, pensar de la existencia de cierta duplicidad de servicios en el territorio.

Para dar respuesta a la última cuestión planteada, es necesario considerar la alineación de las políticas públicas de incubadoras a nivel territorial y la colaboración entre las propias incubadoras. Se hace evidente, pues, la necesidad de reorganizar los recursos a nivel territorial orientados al cliente, considerando la nueva panorámica de crisis económica y social.

Una posible vía de solución al problema sería considerar la creación de una red de incubadoras a nivel territorial, basada en competencias y capacidades de las incubadoras a nivel de servicios y recursos. Con la creación de la red de incubadoras territorial, el cliente, es decir, el emprendedor, podría fácilmente identificar o ser orientado hacia los servicios bá- 
sicos y o avanzados más adecuados para su perfil como empresario y de empresa en las diferentes etapas de la incubación.

De esta manera, cada incubadora de empresas se focaliza en la prestación de los servicios en los que destaca por tener más experiencia o competencia en el territorio, se especializa en una topología de servicio o de sector, y a su vez evita las duplicidades de servicios básicos ofrecidos en el territorio al emprendedor. La red, gestiona pues, la prestación de servicios al emprendedor y a su vez identifica nuevas oportunidades de colaboración con otros agentes económicos, como pudieran ser, las empresas de capital riesgo, para así, cubrir las demandas de servicios no cubiertas en este momento.

La prestación de servicios avanzados, requiere probablemente de la colaboración con una institución académica universitaria, con capacidades de ofrecerle a los emprendedores servicios avanzados de investigación, patentabilidad e innovación. Se espera que, con la creación de una red de incubadoras, la prestación de servicios al emprendedor se realice por capacidades, con independencia de la localidad en la que esté situada la incubadora, y por tanto, se alcance una mejora del servicio recibido que pudiera repercutir en mejores tasas de de retorno económico y social.

\section{Referencias Bibliográficas}

Allen, David N. y Syedur, Rahman (1985). Small Business Incubators: a positive environment for entrepreneurship, Journal of Small Business Management, No. 23, Estados Unidos pp, 12-22.

Allen, David N. y McCluskey, Raymond (1990). "Structure, policy, service and perfor- mance in the business incubator industry", Journal of Entrepreneurship Theory and Practice, Vol. 15, № 2 Estados Unidos, pp 61-67.

Alonso, José María y Fageda, Xavier (2007). Incubadores d'empreses: un instrument de suport a l'emprenedoria amb bona salut a Catalunya., Revista Econòmica de Catalunya, №. 55, España, pp. 32-44.

Baldini, Nicola (2010). University spin-off and their environment. Technology Analysis \& Strategy Management, No. 22, Estados Unidos, pp. 859-876.

Birch, D.L. (1979). The Job Generation Process, MIT Programme on Neighbourhood and Regional Change. Camb. Mass.

Bruneel, Johan; Ratinho, Tiago; Clarysse, Bart y Groen, Aard (2012). The Evolution of Business Incubators: comparing demand and supply of business incubation services across different incubator generations" Technovation, No. 32, Estados Unidos, pp, 110-121.

Druilhe, Céline y Garnsey, Elisabeth (2004). Do academic spin-outs differ and does it matter? The Journal of Technology Transfer, No. 29, Alemania, pp 269-285.

Dvir, Dov; Sadeh, Arik, y Malach-Pines, Ayala (2010). The fit between entrepreneurs' personalities and the profile of the ventures they manage and business success: An exploratory study, Journal of High Technology Management Research, No. 21, Estados Unidos, pp 43-51.

Eisenhardt, Kathleen y Schoonhoven, Claudia (1990). Organizational growth - linking founding team, strategy, environment, and growth among United-States semiconductor ventures, 19781988, Administrative Science Quarterly, No. 35, Estados Unidos, pp 504-529. 
Valorando los programas de apoyo al emprendedor en las incubadoras de empresas... Cerdán-Chiscano, Mónica; Jiménez-Zarco, Ana Isabel y Torrent-Sellens, Joan

Global Entrepreneurship Monitor (GEM 2012). available on-line: http://www.gemconsortium.org/docs/2645/gem-2012global-report.

Hytti, Ulla y Maki, Katja (2007). Which firms benefit most from the incubators? International Journal of Entrepreneurship and Innovation Management. No. 7, pp. 506-523.

Jo, Hyungrae; Lee, Jinjoo (1996). The relationship between and entrepreneur's background and performance in a new venture, Technovation, № 16. Estados Unidos, pp. 161-171.

Mosey, Simon; Wright, Mike, y Clarysse, Bart (2012). Transforming traditional university structures for the knowledge economy through multidisciplinary institutes. Cambridge Journal of Economic, Reino Unido, pp 1-21.

Muhamad Abduh, Clare D'Souza, Ali Quazi, Henry T. Burley (2007). "Investigating and classifying clients' satisfaction with business incubator services", Managing Service Quality, Vol. 17 Iss: 1, pp. 74-91.

Nueno, Pedro (1996). Emprendiendo. Colección Expansión. Ediciones Deusto.

Parker, Simon C. y van Praag, C. Mirjam (2012). 'The entrepreneur's mode of entry: business takeover or new venture start?'. Journal of Business Venturing, vol 27, no. 1, pp. 31-46.

Peña-Vinches, J.C; Bravo, S.; Alvarez, F.A.; y Pineda, D. (2011). "Analysis of characteristic of business Incubators in Colombia: A case Study". Journal of Economics, Finance and Administrative Science, 16(30); pp 13-29.

Peterson, Suzanne J. (1985). Creating jobs by creating businesses: the role of business incubators. National Council for Urban Economic Development. Washington DC.

Potts, Tavis (2010). The natural advantage of regions: linking sustainability, innova- tion and regional development in Australia. Journal of Cleaner Production, No.18, pp 713-725.

Rauch, Andreas y Frase, Michael (2005). "Psychological approaches to entrepreneurial success: A general model and an overview of findings" In C.L. Cooper \& I.T. Robertson (Eds.), International Review of Industrial and Organizational Psychology, pp. 101142. Chichester: Willey.

Schneider, Cédric y Veugelers, Reinhilde (2010). "On young highly innovative companies: Why they matter and how (not) to policy support them". Industrial and Corporate Change, No. 19, pp. 969-1007.

Schwartz, Michael (2012). "Incubation time, incubator age, and firm survival after graduation". International Journal of Entrepreneurship \& Innovation Management; No. 15, pp 108-130.

Schwartz, Michael y Hornych, Christoph. Specialization as strategy for business incubators: An assessment of the Central German Multimedia Center. Technovation. No. 28, pp. 436-449.

Smilor, Raymond W. y Gill, Michael Doud (1986). The new business incubator: Linking talent, technology, capital and Know-how. Lexinton Books.

Torrent, Joan y Vilaseca, Jordi (2008). "TIC, conocimiento y productividad del trabajo. Un ejercicio de descomposición de la eficiencia sectorial de la economía española a partir del análisis Input/Output" a Nuevas tecnologías, nuevos mercados de trabajo / coord. Díaz-Chao, Madrid: Mundi Prensa. P. 173-223.

Ucbsasaran, Deniz, Westhead, Paul y Wright, Mike (2009). The extent and nature of opportunity identification by experienced entrepreneurs. Journal of Business Venturing. No. 24, pp 99-115. 
Urbano, David, y Veciana, José María (2001). "Marco institucional formal de la creación de empresas en Cataluña: oferta y demanda de servicios de apoyo", Documento de Trabajo $n^{\circ}$ 2001/9, XI Congreso Nacional de ACEDE, Barcelona, pp. 22-24 de Septiembre.

Withers, Michael. C; Drnevich, Paul Louis y Marino, Louis (2011). "Doing more with less: The disordinal implications of Firm age for leveraging capabilities for Innovation activity", Journal of Small Business Management, Estados Unidos, No. 49, pp. 515-536.

Wichitchany, Wannapa, Durongwatana, Supol, Vadhanasindhu, Pakpachong (2012). "The factors of innovative organization: some evidences in Thailandia". Global Conference on Business and Finance Proceedings, Estados Unidos, No. 7, pp 531-536. 